\title{
A Neural Network Approach for Locating Multiple Defects
}

\author{
S.J. Farley ${ }^{1, a}$, J.F. Durodola ${ }^{1, b}$, N.A. Fellows ${ }^{1, c}$ and L.H. Hernández-Gómez ${ }^{2, d}$ \\ ${ }^{1}$ Oxford Brookes University, School of Technology, Wheatley Campus, Oxford OX33 1HX, UK \\ ${ }^{2}$ Instituto Politécnico Nacional, ESIME SEPI Edificio 5, 3er piso, Unidad Profesional Adolfo López \\ Mateos, colonia Lindavista 07738 México, D. F. México \\ asfarley@brookes.ac.uk, bjdurodola@brookes.ac.uk, nafellows@brookes.ac.uk, \\ duishector56@hotmail.com
}

Keywords: Lamb wave ultrasound, artificial neural networks, finite element analysis, non destructive evaluation.

\begin{abstract}
A method is presented to demonstrate the use of artificial neural networks (ANNs) in providing additional information regarding defects or flaws when used in conjunction with the ultrasonic A-scan method. ANNs were employed both as pattern classifiers and as function approximators to maximise the amount of data available from the temporal A-scan signal. A steel bar was modelled in 2D using ABAQUS finite element analysis (FEA) software. A single defect was introduced to the bar, modelled as a void, and parametric studies conducted to record data with the defect at various locations. An ultrasonic Lamb wave was introduced at the top of the bar. The longitudinal wave propagated along the length of the bar and was partially reflected by the defect. Multiple cases were simulated, modelling voids between $1 \mathrm{~mm}$ and $6 \mathrm{~mm}$ in width in various locations. Mean displacement of all the nodes at the top of the bar was recorded throughout the simulation, and features extracted from this waveform to create the data set for the ANNs. The ANNs were trained with a percentage of the data collected, selected at random, and assessed with the remaining data. The target data for the ANNs were the depth and size of the defect. The case of two separate defects was also investigated. The procedure was carried out in the same manner as for one defect, but in this case the target data for the ANNs were the depth of the first defect and the distance between the defects. A separate ANN was employed as a pattern classifier, to determine if the reflected A-scan signal represented one or two defects. The final system was tested using previously unseen data, and provided very good results both in determining the number of defects and the size and location of the defects, even with data to which noise had been added.
\end{abstract}

\section{Introduction}

Ultrasonic A-scan inspection has been used extensively over the last few decades to assess components for flaws or to determine component thickness. However, signals generally need to be interpreted by a skilled operator, and only limited information is given in the temporal signal displayed on the user's screen [1]. The use of artificial neural networks to glean the maximum amount of information from a temporal A-scan signal is a topic that has had much attention, but previous studies have generally used ANNs to either classify defects into certain groups [2,3], or to provide a more precise measure of a defect's size and location [4] This study demonstrates the use of multiple ANNs to analyse an A-scan signal from a steel bar containing either one or two defects, and classify the signal into a category ( 1 defect, 2 defects), then to assess the size of the defects and provide detailed information regarding their location. The inverse approach was used, as in a previous work conducted by Hernández-Gómez et al [5] where the ANNs were trained using data from finite element simulations of ultrasonic inspection. This approach saves a great deal of time, as parametric studies can assess many hundreds of cases without the need to prepare hundreds of test specimens. The current study builds on the findings of this past work by creating a simulation that more accurately represents an experimental procedure, and that can therefore be much more easily validated by experiment. 


\section{Finite element analysis}

A steel bar was modelled in two dimensions using ABAQUS finite element analysis software, as shown in Fig. 1. An ultrasonic pulse with a frequency of $100 \mathrm{kHz}$ was applied to the top of the bar as pressure following a sinusoidal function as presented by Yagawa and Okuda [4], although in this case two periods of the sine function were used [6,7]. The maximum amplitude of the pulse was 10MPa. The Young's modulus, Poisson's ratio and density were taken as $210 \mathrm{GPa}, 0.3$ and $7800 \mathrm{~kg} / \mathrm{m}^{3}$ respectively. The material was assumed to be homogeneous and isotropic. Defects were modelled as voids, with height $1 \mathrm{~mm}$ and width $1 \mathrm{~mm}$ to $7 \mathrm{~mm}$. The maximum element size was chosen as $1 \mathrm{~mm}$, to exceed the lower threshold suggested by Lowe and Diligent [7] of at least eight elements per wavelength. The timesteps in the simulations were taken as $0.1 \mu \mathrm{s}$. The longitudinal velocity of an ultrasonic wave is dependent on the geometry of the component and the wavelength of the applied wave, with lower velocity in thin bars and rods [9]. For a bar of width less than one fifth of the wavelength, the velocity of the longitudinal wave is given in Eq. 1 as

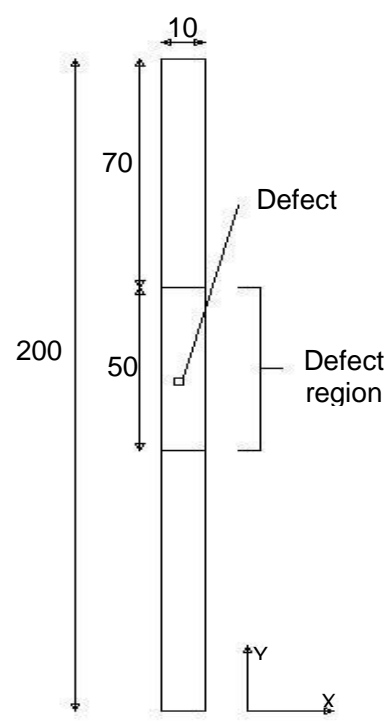

Fig. 1: Diagram of bar (dimensions in $\mathrm{mm}$ )

$$
c=\sqrt{\frac{E}{\rho}},
$$

where $\mathrm{c}$ is the velocity of the dilatational wave in $\mathrm{m} / \mathrm{s}, \mathrm{E}$ is the Young's modulus and $\rho$ is the material's density. Care was taken in the study to minimise the dispersion of the wave as much as possible. To achieve this, a frequency thickness product of $1 \mathrm{MHz} . \mathrm{mm}$ was chosen, meaning that only the primary compressional (symmetric) wave $\mathrm{s}_{0}$, and the primary flexural (antisymmetric) wave $\mathrm{a}_{0}$ would be present [10]. The input frequency of the ultrasonic pulse was therefore $100 \mathrm{kHz}$. This made the received signal much easier to read and improved the accuracy of data presented to the ANNs.

\section{Feature extraction}

In order to train the ANNs, it was necessary to create a data set using representative features of the wave response measured at the top of the bar. For this study, the wave response from a bar with no defect was subtracted from the wave response from the bar with a defect, so that the resultant wave showed the difference between signals, a method demonstrated by Yagawa and Okuda [4]. From this signal, in the case of a single defect, the time and the amplitude of the first major peak were taken as features, as shown in Fig. 2, and used to train and subsequently assess the ANNs. The time of the peak corresponded to the distance to the defect (depth) and the height of the peak corresponded to the size of the defect (magnitude). Consequently, each defect position was represented by just two values, meaning that training and assessment of the ANNs in this case was much faster than in previous work [5]. In the case of two defects, more data were required to accurately train the ANNs, and so the time and height of up to seven peaks, starting with the first positive peak, were used to represent the signal. This resulted in up to 14 data values for each case. Fig. 3 shows four examples of the signal from a bar with two defects, and it can be seen that the representative waveform has either three, four or five positive peaks depending on the distance between the defects. Of particular interest were cases where the two defects were less than a quarter of a wavelength apart. In these cases, the two separate reflected sinusoidal waves combined to form a slightly elongated wave of a similar form to that expected from a single defect, and so the additional information provided by recording every peak was necessary for the classifier ANN to 
accurately distinguish between single and double defect cases. All data were normalised before being presented to the ANNs, to ensure that the data lay in the range $[-1,1]$. This was done by dividing each value by the maximum value for the data set.
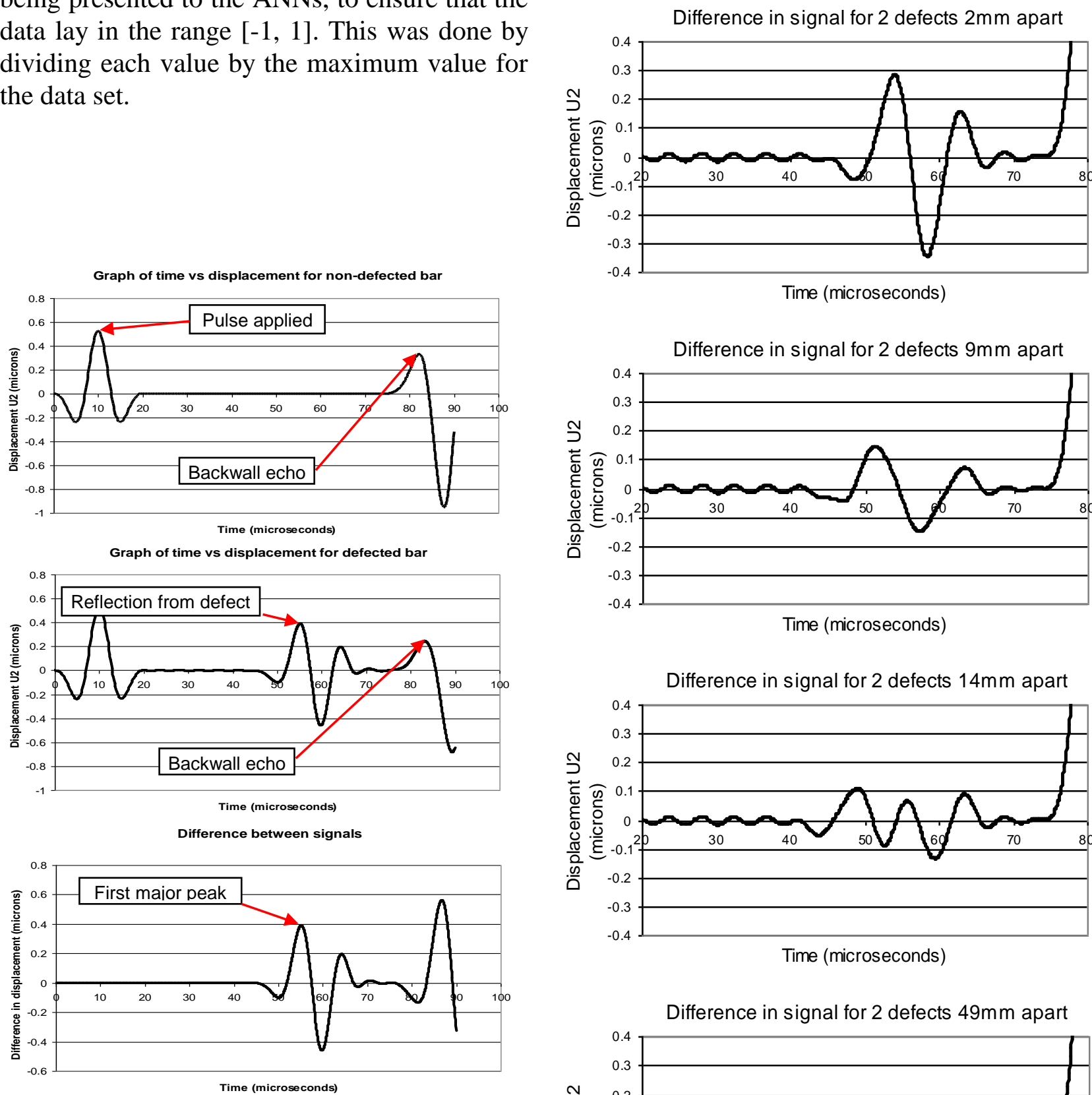

Fig. 2: Ultrasonic wave response for feature extraction

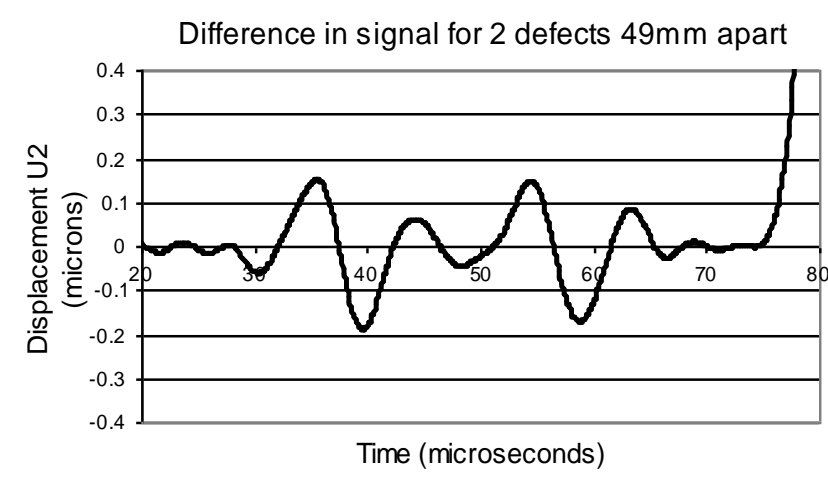

Fig. 3: Difference in signal for selected 2 defect cases

\section{Artificial neural networks}

An artificial neural network (ANN) is essentially a mathematical model aimed to function like the neural network in the human brain, and is able to map input data to the appropriate output data. ANNs are often used as pattern classifiers or as function approximators, and in this study both types were employed. The ANNs used were of the feedforward multilayer perceptron type, and all had 3 layers, as illustrated in Fig. 4. 

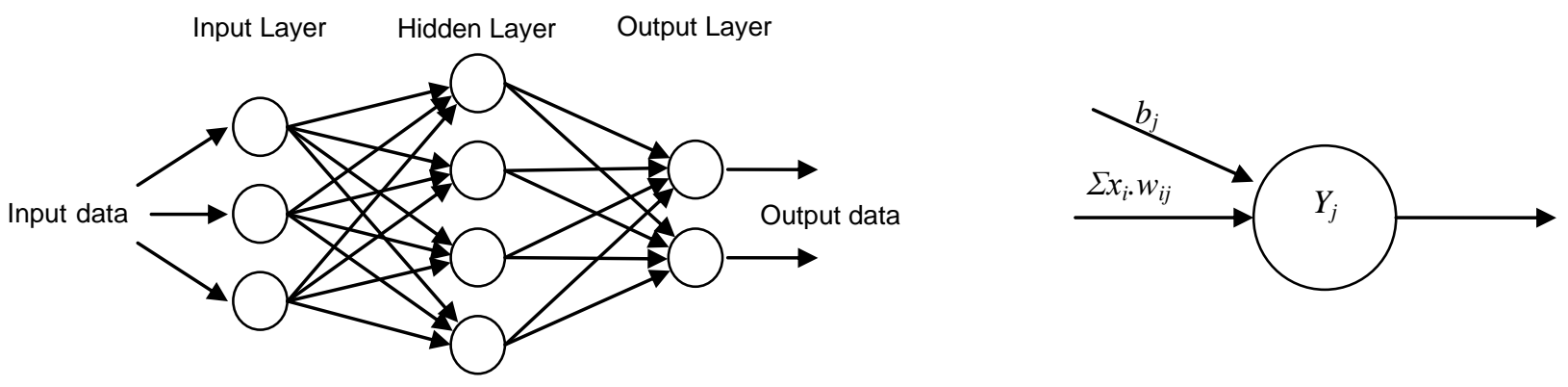

Fig. 4: A typical three layer ANN

Fig. 5: A perceptron and its activation function

Each perceptron performs a simple mathematical function but linked together a network of perceptrons can perform very complex functions e.g. to approximate any nonlinear function. The input to each neuron is illustrated in Fig. 5, and is given as

$$
y_{-} i n_{j}=b_{j}+\sum_{i=0}^{n} x_{i} w_{i j}
$$

where $y_{-} i n_{j}$ is the net input to a neuron $Y_{j}, b_{j}$ is the bias on unit $Y_{j}, x_{i}$ is the signal from the previous neuron and $\mathrm{w}_{\mathrm{ij}}$ the weight between connected neurons. In the multilayer perceptron network, each neuron in one layer is connected to every neuron in the next layer, hence the summation of the signals in Eq. 3. In the normal operation of the network, data travel in the forward direction only, hence the term feedforward. Once the input signal arrives at each neuron, a transfer function is applied to it, and the resultant value is passed on to the next neuron in the network. In this case, the first and second layers of the network used a Tan-Sigmoid function, whose equation is given as

$$
f(x)=\frac{2}{1+\exp (-\sigma x)}-1
$$

where $\sigma$ is a parameter to alter the shape of the curve. The sigmoid function is desirable in the perceptron model as it ensures that all values passing to the next neuron lie in the range $[-1,1]$. The output layer used a linear transfer function, to ensure that erroneous outputs were easy to identify. All three ANNs used in this study had 20 neurons in the input layer, 40 neurons in the hidden layer and either one or two neurons in the output layer, depending on their application. Before an ANN can be used, it needs to be trained, which is achieved by creating a data set of inputs and corresponding target outputs. Initially, the weights and biases in the network are set to random values. All data is passed through the ANN and the output compared to the target. The weights and biases in the network are modified based on the amount of error, and the process is repeated. This is known as back-propagation, and continues until either a predetermined number of epochs is reached, or a satisfactory level of convergence is attained. For this study, all networks were trained for 20,000 epochs with a convergence target of zero error using the Neural Network toolbox in MATLAB 7.0. Training was carried out using the scaled conjugate gradient (SCG) method [11] using $90 \%$ of the available data selected at random. The remaining data were used to assess the ANNs. In order to assess the trained ANNs' ability to deal with noisy or incomplete data, noise was added to the assessment data set using the formula presented by Sahin and Shenoi [12], shown below in its modified format to increase the range to $\left[\mathrm{V}_{\mathrm{o}}-\mathrm{n}, \mathrm{V}_{\mathrm{o}}+\mathrm{n}\right]$.

$$
V_{n}=V_{o} \times[1+(R A N D-0.5) .(2 n)],
$$


where $\mathrm{V}_{\mathrm{n}}$ is the value with noise added, $\mathrm{V}_{\mathrm{o}}$ is the original value, RAND represents a random number between 0 and 1 , and $n$ is the amount of noise to be added. Noise was added to all data points in the assessment set (a different value of RAND for each point) ten times, and a mean value taken for each point before being presented to the ANNs.

\section{Results}

The trained ANNs were presented with data they had not previously seen to test their ability to deal with new data, and also presented with the same previously unseen data to which artificial noise had been added, to simulate experimental data. The first ANN was employed as a pattern classifier, and indicated whether the case presented had either one or two defects, based on the 14 data points representing the ultrasonic signal. The correlation was very good in this case, with over $99 \%$ correct classification in the case without noise, and $93 \%$ correct with $15 \%$ added noise, as shown in Table 1.

Accuracy of ANN classification into one or two defect cases

\begin{tabular}{|c|c|c|c|c|c|}
\hline Amount of noise added [\%] & 0 & 5 & 10 & 15 & 20 \\
\hline Correctly classified cases [\%] & 99.17 & 96.69 & 93.39 & 93.39 & 89.26 \\
\hline
\end{tabular}

Table 1: ANN classification accuracy

After the first ANN had classified the cases, the data were then transferred to the appropriate second ANN, which would either give a value for depth of defect and size of defect in the case of a single defect, or a value for depth of defect and distance to the second defect in the case of two defects. As can be seen from Fig. 6, predictions of defect location and size for the case of a single defect correlate excellently with actual values. The reduction in accuracy of prediction was only slightly degraded when up to $15 \%$ error noisy data was added to true values and predictions re-analysed.
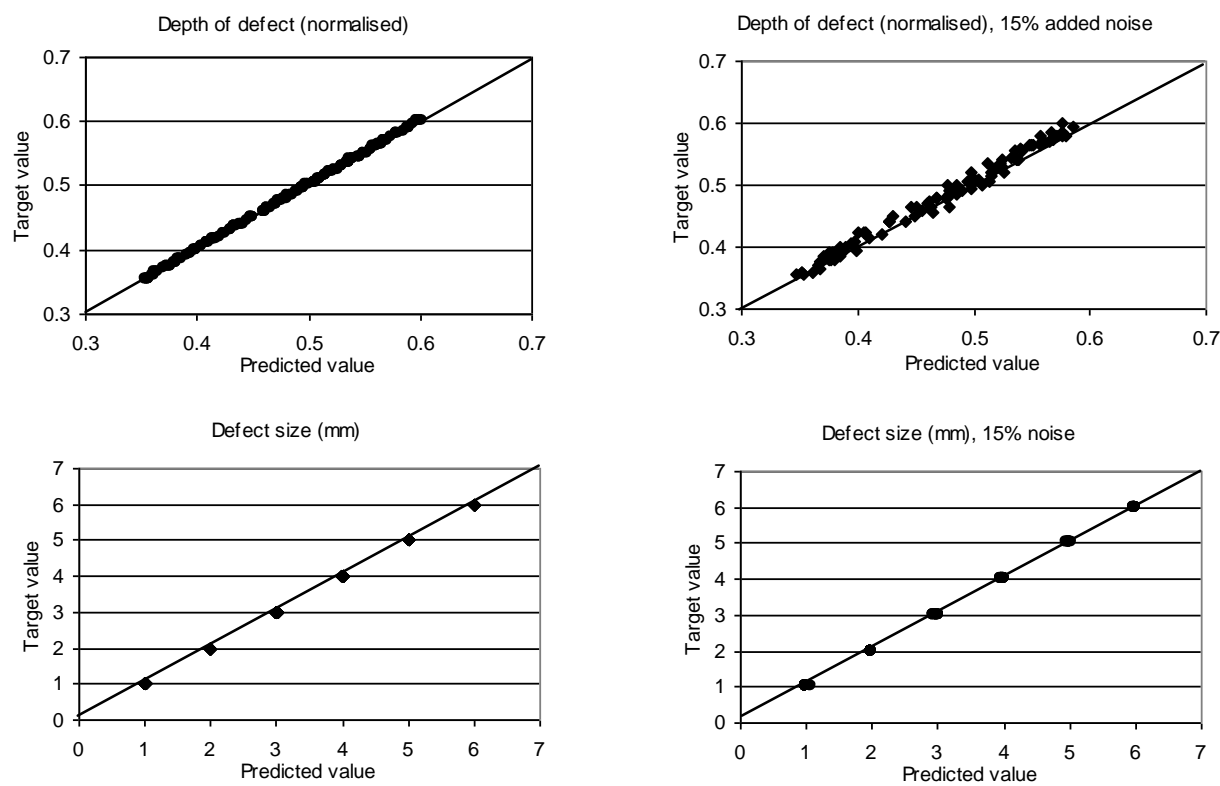

Fig. 6: Comparison of prediction of location and size of defect with target values for the case of single randomly placed defects. 
Fig. 7 shows results of analysis of location for the cases with two defects. As with the single defect case, the predicted location of the first and second defects correlate excellently with actual values, and a strong trend towards actual values is observed with $5 \%$ noise added to the test data. The correlation was seen to deteriorate when more than about $5 \%$ of noise was added. It is likely that separate ANNs to determine the depth of the first defect and the distance between the defects would reduce this error, and this will be considered in later studies.
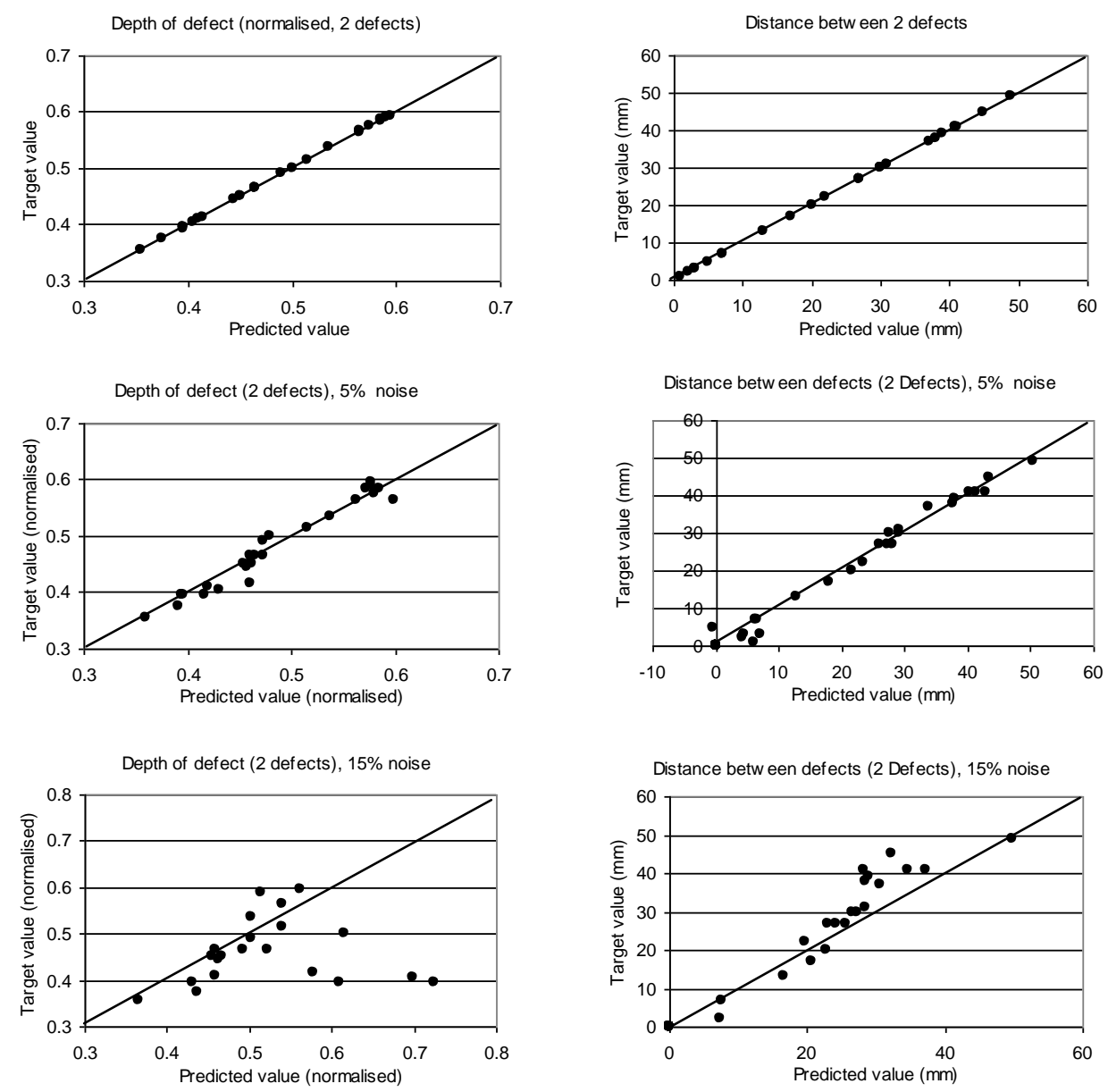

Fig. 7: Comparison of prediction of location of defects with target values for the case of two randomly placed defects

\section{Conclusions}

A method has been presented that can classify an ultrasonic A-scan signal according to the number of defects present, and then provide an accurate measure of the size and location of the defect(s). The predictions correlate very well with target values, even with noisy data, and it is expected that the accuracy of the ANNs' predictions could be improved with further refinement in feature extraction and network design. Future work will include presenting trained ANNs with experimental data, investigating the effect of multiple defects of different sizes and investigating more complex component geometry. 


\section{References}

[1] Achenbach, J D (2000) Quantitative non-destructive evaluation. International Journal of Solids and Structures. 37, 13-27.

[2] Veiga, J L B C, De Carvalho, A A, Da Silva, I C and Rebello, J M A (2005) The use of artificial neural network in the classification of pulse-echo and TOFD ultra-sonic signals. J. of the Braz. Soc. of Mech. Sci. \& Eng. Vol. XXVII, No. 4, 394-398.

[3] Legendre, S, Massicotte, D, Goyette, J and Bose, T K (2001) Neural classification of lamb wave ultrasonic weld testing signals using wavelet coefficients. IEEE Transactions on Instrumentation and Measurement. Vol. 50, No. 3, 672-678.

[4] Yagawa, G. and Okuda, H. (1996) Neural networks in computational mechanics. Archives of Computational Methods in Engineering. Vol. 3, 4, 435-512.

[5] Hernández-Gómez, L H, Durodola, J F, Fellows, N A and Urriolagoitia-Calderón, G (2005) Locating defects using dynamic strain analysis and artificial neural networks. Applied Mechanics and Materials. Vols. 3-4, 325-330.

[6] Lowe, M J S, Challis, R E, Chan, C W (2000) The transmission of Lamb waves across adhesively bonded lap joints. J. Acoust. Soc. Am. 107 (3), 1333-1345.

[7] Lowe, M J S and Diligent, O (2002) Low-frequency reflection characteristics of the $\mathrm{s}_{0}$ Lamb wave from a rectangular notch in a plate. J. Acoust. Soc. Am. 111 (1), 64-74.

[8] Barauskas, R and Daniulaitis, V (2000) Simulation of the ultrasonic wave propagation in solids. Ultragarsas. No. 4 (37), 34-37

[9] Brown, A E. Rationale and summary of methods for determining ultrasonic properties of materials at Lawrence Livermore National Laboratory. National Technical Information Service, Sprigfield, VA, 1997.

[10]Rose, J L. Ultrasonic waves in solid media. Cambridge University Press, 1999.

[11]Demuth, H. and Beale, M. Neural Network Toolbox for use with MATLAB, The MathWorks, 2001.

[12] Sahin, M and Shenoi, R A (2003) Vibration-based damage identification in beam-like composite laminates by using artificial neural networks. Proc. Instn. Mech. Engrs. Vol. 217 Part C, 661-676 